\title{
PERFIL ANTROPOMÉTRICO E DE CONSUMO ALIMENTAR DE JOGADORES VIRTUAIS DE LEAGUE OF LEGENDS (JOGOS ELETRÔNICOS)
}

\author{
ANTHROPOMETRIC PROFILE AND FOOD CONSUMPTION OF \\ VIRTUAL PLAYERS OF LEAGUE OF LEGENDS (ELECTRONIC \\ GAMES)
}

\author{
Dias, IRR ${ }^{1}$, Silva, GC ${ }^{2}$, Cardoso, $\mathrm{LGV}^{3}$, França, $\mathrm{VF}^{4}$, Sousa, $\mathrm{LFB}^{5}$, Rocha, $\mathrm{LS}^{6}$ \\ Afiliações: 1- Nutricionista graduado pela Universidade Federal da Bahia, Instituto Multidisciplinar em Saúde, Campus Anísio Teixeira, Vitória \\ da Conquista, Bahia, Brasil. \\ 2- Graduanda em Nutrição pela Universidade Federal da Bahia, Instituto Multidisciplinar em Saúde, Campus Anísio Teixeira, Vitória da \\ Conquista, Bahia, Brasil. \\ 3- Nutricionista e Doutor em Saúde Pública, Professor do curso de Nutrição da Universidade Federal da Bahia, Instituto Multidisciplinar em \\ Saúde, Campus Anísio Teixeira, Vitória da Conquista, Bahia, Brasil. \\ 4- Nutricionista e Doutora em Nutrição, Professora do curso de Nutrição da Universidade Federal da Bahia, Instituto Multidisciplinar em Saúde, \\ Campus Anísio Teixeira, Vitória da Conquista, Bahia, Brasil. \\ 5- Nutricionista Doutoranda em Ciências Fisiológicas pela Universidade Federal da Bahia, Instituto Multidisciplinar em Saúde, Campus Anísio \\ Teixeira, Vitoria da Conquista-Bahia. \\ 6-Nutricionista graduado pela Universidade Federal da Bahia, Instituto Multidisciplinar em Saúde, Campus Anísio Teixeira, Vitoria da Conquista, \\ Bahia, Brasil. Mestre em Bioquímica e Biologia Molecular pela Universidade Estadual do Sudoeste da Bahia, Professor do Curso de Nutrição da \\ Faculdade de Tecnologia e Ciências, Campus de Jequié - Bahia. \\ Endereço do autor de correspondência: Rua Hormindo Barros, 58, Candeias, Vitória da Conquista, Bahia, Brasil. CEP: $45029-094$. \\ luizgvc1@gmail.com
}

\section{Resumo}

League of Legends é um jogo eletrônico no estilo Multiplayer Online Battle Arena. O estilo do esporte exige várias horas diárias de treinamento em frente ao computador levando seus praticantes a um estilo de vida sedentária. $O$ presente estudo teve como objetivo avaliar o perfil antropométrico e consumo alimentar de atletas amadores e profissionais de League of Legends. Trata se de um estudo transversal realizado com jogadores da cidade de Vitória da Conquista-BA. Os participantes foram submetidos à avaliação antropométrica e do consumo alimentar. Para a avaliação antropométrica foram mensurados peso, altura, circunferências e dobras cutâneas. Para determinar a ingestão calórica de macronutrientes e micronutrientes, foram utilizados o questionário de frequência alimentar e o registro alimentar. Como resultado, segundo a média do $\operatorname{IMC}\left(25,3 \pm 6.04 \mathrm{~kg} / \mathrm{m}^{2}\right)$ os jogadores apresentavam sobrepeso; a média do percentual de gordura corporal $(\mathbf{1 7 , 9 6 \pm 0 , 7 2 \% )}$ foi classificada como "moderamente alto". Em relação ao padrão de ingestão alimentar, os grupos alimentares dos tubérculos, frutas, castanhas e amêndoas apresentaram as menores taxas de consumo diário; já o registro alimentar identificou inadequações nos três macronutrientes, assim como em alguns micronutrientes. $O$ presente estudo identificou o perfil de sobrepeso nos jogadores e alterações no consumo de macro e micronutrientes.

Palavras-chave: Antropometria; Consumo alimentar; Atletas; League of Legends.

\section{Abstract}

League of Legends is a Multiplayer Online Battle Arena style electronic game. The style of sport requires several hours of training daily in front of the computer, leading its practitioners to a sedentary lifestyle. This study aimed to evaluate the anthropometric profile and food consumption of amateur and professional League of Legends athletes. This is a cross-sectional study carried out with players from the city of Vitória da Conquista-BA. Participants underwent anthropometric and food consumption assessment. For the anthropometric evaluation, weight, height, circumferences and skinfolds were measured. To determine the caloric intake of macronutrients and micronutrients, the food frequency questionnaire and food record were used. As a result, according to the mean BMI $\left(25.3 \pm 6.04 \mathrm{~kg} / \mathrm{m}^{2}\right)$ players were overweight; the mean percentage of body fat $(17.96 \pm 0.72 \%)$ was classified as "moderately high". Regarding the pattern of food intake, the food groups of tubers, fruits, nuts and almonds had the lowest daily consumption rates; the food record identified inadequacies in the three macronutrients, as well as in some micronutrients. The present study identified the overweight profile of players and changes in the consumption of macro and micronutrients. 
Introdução

Os jogos eletrônicos vêm se
desenvolvendo em conjunto com o
desenvolvimento tecnológico. À medida que
novos hardwares e softwares vão surgindo, os
jogos vão se moldando e ganhando destaque na
rotina dos seus adeptos ${ }^{1}$. Nos últimos anos, a
indústria dos videogames vem evoluindo cada
vez mais, sendo uma vertente dessa progressão
os chamados e-Sports, que ainda não tem uma
definição bem elucidada, mas entende-se como
um esporte no qual os jogadores treinam
habilidades físicas e mentais a partir da
tecnologia ${ }^{2}$.

No cenário dos e-Sports é evidente que alguns jogos possuem mais destaque do que outros. Um exemplo que está em ascensão é o League of Legends, um jogo no estilo MOBA ("Multiplayer Online Battle Arena", que significa "arena de batalha online para vários jogadores"), no qual duas equipes com 5 jogadores se enfrentam com o objetivo de conquistar a base adversária ${ }^{3}$.

A partir do momento em que os indivíduos começam a disputar torneios e treinar com seu time deixam de serem jogadores de videogame e passam a serem chamados de $e$ atletas $^{4}$. Todo atleta almeja conquistas e, por isso, eles possuem uma comissão técnica ao seu lado composta por um treinador, um analista estratégico e dois assistentes técnicos, os quais estabelecem metas para que as conquistas possam ser alcançadas ${ }^{5}$.
Como se trata de um esporte no qual os atletas passam a maior parte do dia em frente ao computador praticando suas habilidades cognitivas e motoras no jogo é necessária atenção, pois pessoas com hábitos sedentários associados a uma má alimentação possuem maior risco de desenvolver doenças crônicas não transmissíveis (DCNTs) em relação àqueles que praticam atividade física e possuem hábitos alimentares saudáveis ${ }^{6,7}$. No caso dos e-atletas soma-se um terceiro fator de risco que consiste no estresse gerado pela cobrança durante as competições ${ }^{4}$.

O fato do esporte exigir várias horas diárias de treinamento em frente ao computador leva seus praticantes a um estilo de vida sedentária, levando os jogadores a uma predisposição maior ao sobrepeso ${ }^{8-11}$. Além disso, o esporte exige um alto nível de concentração e pouco tempo livre, o que faz com que os adeptos tenham uma tendência para escolha de alimentos mais práticos, como os processados e ultraprocessados ${ }^{4}$.

Com a popularização dos e-sports, o número de jogadores tem aumentado significativamente. Conforme dados do Esportv $^{12}$, em 2018 na final do campeonato Mundial havia mais de 23 mil pessoas presentes no estádio e 44 milhões de espectadores conectados simultaneamente. Já na final do campeonato Mundial de 2019, o pico de audiência foi de 44 milhões de espectadores simultâneos ${ }^{13}$. Contudo, não é encontrado na literatura estudos que abordam o estado nutricional dos praticantes de League of 
Legends na Bahia. Diante disso, o presente estudo teve como objetivo avaliar o perfil antropométrico e consumo alimentar de atletas amadores e profissionais de League of Legends de uma região do sudoeste baiano.

\section{Métodos}

A amostra foi constituída por jogadores amadores e profissionais do sexo masculino que estavam jogando em campeonatos organizados no shopping center e em universidades particulares da região de Vitória da Conquista, Bahia, Brasil. Os critérios de inclusão no estudo foram: idade igual ou superior a 18 anos, estar jogando nas filas ranqueadas (plataforma de disputa online) por no mínimo 6 meses e ter jogado pelo menos três campeonatos. Presença de lesões impeditivas de participar dos jogos configurou-se como critério de exclusão.

Todos os participantes foram esclarecidos individualmente sobre os objetivos, procedimentos, riscos e benefícios oriundos deste estudo, tendo a opção de recusar ou participar como voluntário, mediante a assinatura do Termo de Consentimento Livre e Esclarecido (TCLE). A pesquisa foi aprovada pelo Comitê de Ética em Pesquisa com Seres Humanos da Universidade Federal da Bahia, Instituto Multidisciplinar em Saúde, sob parecer $n^{\circ} 3.155 .999$.

A coleta de dados foi realizada em um único momento, no laboratório de avaliação nutricional da Universidade Federal da Bahia, campus de Vitória da Conquista. Para a obtenção dos dados referentes às condições socioeconômicas, demográficas e estilo de vida dos participantes realizaram-se entrevistas individuais. Foi utilizado um questionário semiestruturado abordando dados relativos à idade, sexo, renda mensal, histórico de doenças individual e familiar, prática de exercícios físicos e hábitos da rotina diária.

Para a avaliação do consumo alimentar, foi aplicado um registro alimentar prospectivo de três dias (dois registros efetuados em dias da semana e um no final de semana), sendo estes não consecutivos nos quais o participante anotou tudo que ingeriu durante o dia, descrevendo o horário, local, preparação e detalhamento da refeição/alimento e a quantidade em medidas caseiras. Foram fornecidas todas as explicações necessárias para o preenchimento do registro alimentar.

Para complementar a análise qualitativa do consumo alimentar foi aplicado um Questionário Qualitativo de Frequência Alimentar. Foi utilizado um instrumento próprio, preenchido pelos jogadores com acompanhamento dos pesquisadores. As frequências de consumo alimentar foram separadas em cinco opções, a saber: diário, semanal, quinzenal, mensal e nunca. Os alimentos foram ordenados em dez grupos alimentares: laticínios; pães, massas e cereais; tubérculos; leguminosas; ovos, carnes e peixes; frutas; castanhas e amêndoas; óleos e gorduras; açúcares e doces; bebidas e infusões.

Para avaliação do estado nutricional, foram realizadas medidas antropométricas como peso, altura, circunferências e dobras cutâneas. 
A altura $(\mathrm{cm})$ dos atletas foi aferida através de um estadiômetro portátil (Altura exata, escala de 35 a $213 \mathrm{~cm}$ e precisão de $0,1 \mathrm{~cm})$. Para as medidas das circunferências $(\mathrm{cm})$ do braço, cintura e quadril, foi utilizada uma fita métrica inextensível (MacroLife ${ }^{\circledR}$, com graduação de 1 $\mathrm{mm})$. O peso $(\mathrm{kg})$ dos atletas foi aferido utilizando uma balança mecânica (Welmy, aprovada pelo Inmetro, com capacidade de 150 $\mathrm{kg}$ e precisão de $100 \mathrm{~g}$ ). Tais medidas foram realizadas de acordo com as recomendações do Sistema de Vigilância Alimentar e Nutricional $(\text { SISVAN) })^{14}$. Para as dobras cutâneas (bicipital, tricipital, suprailíaca e subescapular) foi utilizado o plicomêtro cientifico (Cescorf com sensibilidade de $0,01 \mathrm{~mm}$ ), as dobras foram aferidas em triplicata e utilizada a média delas, sempre do lado direito do atleta e pelo mesmo avaliador em todas as ocasiões.

Para caracterização do estado nutricional foi utilizado o Índice de Massa Corporal (IMC), utilizando como pontos de corte valores menores que $18,5 \mathrm{~kg} / \mathrm{m}^{2}$ para desnutrição, de 18,5 a $24,9 \mathrm{~kg} / \mathrm{m}^{2}$ para eutrofia, de 25 a $29,9 \mathrm{~kg} / \mathrm{m}^{2}$ para sobrepeso e maiores que $30 \mathrm{~kg} / \mathrm{m}^{2}$ para obesidade, assim como preconiza a Organização Mundial da Saúde para adultos ${ }^{15}$. Para calcular a densidade corporal $\left(\mathrm{g} . \mathrm{cm}^{-3}\right)$ foi utilizado o protocolo de quatro dobras de Durnin $\&$ Womersley $^{16}$. A determinação do percentual de gordura foi realizada através da utilização da equação de $\operatorname{Siri}^{17}$. Para a classificação do percentual de gordura $(\% \mathrm{~g})$ foi utilizado os pontos de corte propostos por Pollock e Wilmore $^{18}$ de acordo com o sexo e faixa etária dos atletas, onde os valores $<4$ indicavam $\% \mathrm{~g}$ muito baixo, de 4 a 6 excelente, de 7 a 10 muito bom, de 11 a 13 bom, de 14 a 16 adequado, de 17 a 20 moderamente alto, de 21 a 24 alto e $>24$ muito alto.

Por meio da densidade corporal foi obtido os valores de massa magra ( $\mathrm{MM} \mathrm{kg}$ ) e massa gorda (MG kg). Para o cálculo da AMB foram utilizadas as equações citadas por Gurney \& Jelliffe ${ }^{19}$.

Para avaliação da composição nutricional das refeições consumidas, utilizou-se o software Dietbox, e os macronutrientes foram convertidos em quilocalorias (kcal) sendo que cada grama de carboidrato/proteína foi equivalente a $4 \mathrm{kcal}$; cada grama de lipídeo a 9 kcal, obtendo-se desta forma o valor energético das refeições diárias. Para determinar a necessidade calórica, foi utilizada a fórmula da Food and Agriculture Organization (FAO) ${ }^{20}$ considerando o peso atual do jogador e utilizando o fator atividade física a 1.2 (sedentário) referente ao estilo de vida referido pelos mesmos.

Para avaliar a adequação dos macronutrientes foram utilizados intervalos de distribuição de macronutrientes para adultos (Acceptable Macronutriente Distribuition Range - AMDR) propostos pelo Institute of Medicine $^{21}$, que preconiza a oferta de carboidratos na distribuição entre $45 \%$ a $60 \%$, de proteínas entre 10 e 35\% e lipídios de 20 a 35\% do valor energético total (VET). Os micronutrientes foram analisados utilizando como referência as Dietary reference intakes $(\text { DRIs })^{22}$. 
Os dados foram tabulados em planilhas do Excel versão 2013 e a análise realizada por meio do software Epi Info versão 3.5.2. As variáveis quantitativas foram apresentadas em médias e desvio padrão e as variáveis categóricas em valores percentuais.

\section{Resultados}

No presente estudo, foram avaliados 28 participantes do sexo masculino, com idade média de $21,53 \pm 2,74$ anos. Na tabela 1 são apresentadas as variáveis antropométricas dos participantes

Tabela 1. Características antropométricas dos jogadores de League of Legends (plataforma online) de Vitória da Conquista, Bahia, Brasil $(\mathrm{n}=28)$.

\begin{tabular}{|c|c|c|c|c|}
\hline Variáveis & Média & DP & Mínimo & Máximo \\
\hline Idade (anos) & 21.53 & 2.74 & 18 & 29 \\
\hline Estatura $(\mathrm{cm})$ & 176.63 & 0.06 & 164 & 190 \\
\hline Peso corporal (kg) & 79.26 & 19.95 & 45.6 & 145 \\
\hline $\operatorname{IMC}\left(\mathrm{kg} / \mathrm{m}^{2}\right)$ & 25.31 & 6.04 & 16.4 & 49.6 \\
\hline Gordura corporal $(\%)$ & 17.96 & 0.72 & 8.6 & 34.9 \\
\hline MG (kg) & 14.88 & 8.32 & 4.8 & 39.8 \\
\hline $\mathrm{MM}(\mathrm{kg})$ & 64.42 & 13.5 & 40.8 & 105.2 \\
\hline $\operatorname{AMB}\left(\mathrm{cm}^{2}\right)$ & 54.63 & 16.5 & 20.7 & 62.2 \\
\hline \multicolumn{5}{|l|}{ Dobras cutâneas (mm) } \\
\hline Tricipital & 12.86 & 7.58 & 7.5 & 25.6 \\
\hline Bicipital & 6.51 & 3.46 & 3.1 & 10.8 \\
\hline Subescapular & 15.01 & 7.89 & 7.9 & 37.8 \\
\hline Supra ilíaca & 16.29 & 8.84 & 6.6 & 25.7 \\
\hline$\Sigma 4 \mathrm{DC}$ & 50.51 & 25.35 & 21.4 & 102.1 \\
\hline \multicolumn{5}{|l|}{ Perímetros (cm) } \\
\hline Braço & 29.95 & 4.29 & 18.5 & 36 \\
\hline Cintura & 82.73 & 12.1 & 64.8 & 117 \\
\hline Quadril & 98.21 & 11.7 & 69.3 & 136 \\
\hline
\end{tabular}

$\mathrm{DP}=$ desvio-padrão; $\mathrm{IMC}=$ Índice de massa corporal; $\mathrm{MG}=$ Massa de gordura; $\mathrm{MM}=$ massa magra; $\mathrm{AMB}=$ área muscular do braço; $\Sigma$ 4DC= somatório das 4 dobras cutâneas.

No gráfico 1 é apresentada a classificação quanto ao percentual de gordura dos jogadores. Apesar da média de IMC (25,31 $\left.\pm 6,04 \mathrm{Kg} / \mathrm{m}^{2}\right)$ ter sido classificada como sobrepeso (tabela 1), 18 dos jogadores apresentaram percentual de gordura classificados como muito bom (7 a $10 \%)$, bom (11 a 13\%) e excelente (14 a 16\%). 
Gráfico 1. Classificação do percentual de gordura para os jogadores de League of Legends (plataforma online) de Vitória da Conquista, Bahia, Brasil segundo Pollock \&Willmore (1993) $(n=28)$.

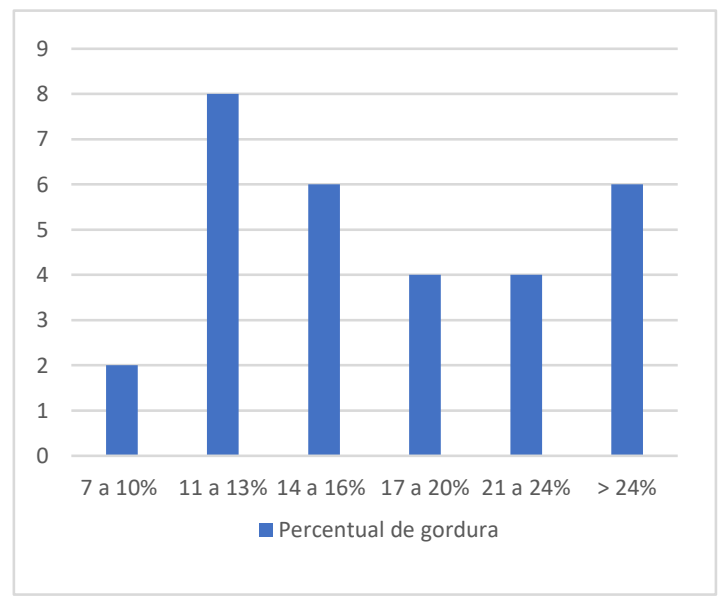

Os dados referentes ao gasto energético, ingestão calórica e macronutrientes dos jogadores de League of Legends, estão expressos na tabela 2 .

Tabela 2. Gasto energético, ingestão calórica e ingestão de macronutrientes pelos jogadores de League of Legends (plataforma online) de Vitória da Conquista, Bahia, Brasil. ( $\mathrm{n}=18)$

\begin{tabular}{c|c|c|c|c|c|c}
\hline Variáveis & $\begin{array}{c}\text { Recomendações } \\
\text { diárias (Homens } \\
\text { Mulheres) }\end{array}$ & Média & $\begin{array}{c}\text { Média } \\
\text { em \% }\end{array}$ & DP & Mínimo & Máximo \\
\hline Gasto energético (cal) & - & 2206,9 & - & 390 & 1655 & 3071 \\
Ingestão Diária (cal) & - & 1834,2 & - & 732,4 & 962,3 & 3331,3 \\
Proteínas (g/\%) & $10-35 \%$ & 85,5 & 17,8 & 53,6 & 27,4 & 229,5 \\
Carboidratos (g/\%) & $45-65 \%$ & 190 & 44,2 & 74 & 102,7 & 373,7 \\
Lipídeos (g/\%) & $20-35 \%$ & 80,2 & 38,9 & 43,6 & 27,2 & 195,9 \\
\hline
\end{tabular}

$\mathrm{DP}=$ desvio-padrão; cal= calorias diárias; $\mathrm{g}=$ gramas; $\%=$ percentual.

A tabela 3 traz a avaliação do consumo de micronutrientes dos jogadores. Ao comparar os valores de recomendação diária aos valores da média de ingestão dos jogadores, é possível verificar que dentre os micronutrientes avaliados, magnésio, ferro, selênio, fósforo e as vitamina E, C, B1, B3, B6, B9 e B12 apresentam valores de ingestão abaixo da recomendação.

Tabela 3. Avaliação do consumo de micronutrientes pelos jogadores de League of legends (plataforma online) de Vitória da Conquista, Bahia, Brasil. ( $\mathrm{n}=10)$

\begin{tabular}{l|c|c|c|c|c|c}
\hline \multicolumn{1}{c|}{ Variáveis } & $\begin{array}{c}\text { Recomendações } \\
\text { diárias } \\
\text { (RDA/AI) }\end{array}$ & $\begin{array}{c}\text { \% } \\
\text { Adequação }\end{array}$ & Média & DP & Mínimo & Máximo \\
\hline Zinco (mcg) & 11 & 80,91 & 8,9 & 5,3 & 4,1 & 21,5 \\
Magnésio (mcg) & 400 & 43,18 & 172,7 & 131,1 & 65,1 & 549,6 \\
Sódio (mcg) & 1500 & 154,01 & 2310,2 & 716 & 1437,2 & 3549,3 \\
\hline
\end{tabular}




\begin{tabular}{l|c|c|c|c|c|c}
\hline Ferro (mg) & 8 & 83,75 & 6,7 & 1,1 & 4,6 & 8,7 \\
Selênio (mcg) & 55 & 77,82 & 42,8 & 7,2 & 29,3 & 54,3 \\
Fósforo (mg) & 700 & 58,92 & 412,7 & 130,9 & 236,8 & 612,7 \\
Vitamina A (mcg) & 900 & 100,09 & 900,8 & 985,9 & 121,2 & 3293,1 \\
Vitamina E (mg) & 15 & 90,00 & 13,5 & 9,2 & 1,3 & 25,5 \\
Vitamina C (mg) & 90 & 47,11 & 42,4 & 29,5 & 0,32 & 100,2 \\
Vitamina B1 (mg) & 1,2 & 91,67 & 1,1 & 0,3 & 0,7 & 1,7 \\
Vitamina B3 (mg) & 16 & 8,13 & 1,3 & 0,5 & 0,6 & 2,1 \\
Vitamina B6 (mg) & 1,3 & 69,23 & 0,9 & 0,3 & 0,4 & 1,4 \\
Vitamina B9 (mcg) & 400 & 54,80 & 219,2 & 81,7 & 117,9 & 421,8 \\
Vitamina B12 & 2,4 & 62,50 & 1,5 & 0,4 & 0,7 & 2,1 \\
(mcg) & & & & & \\
\hline DP= desvio-padrão; mcg= microgramas; mg= miligrama.
\end{tabular}

Na tabela 4 é demonstrado a avaliação do consumo dos grupos alimentares pelos atletas. É possível verificar que o grupo dos pães, massas e cereais e dos ovos, carnes e peixes são os mais consumidos diariamente, o consumo é de $100 \%$ e $96,67 \%$ por meio dos jogadores, respectivamente. Em contrapartida o grupo das frutas, castanhas e amêndoas e dos tubérculos obtiveram os menores percentuais de consumo diário.

Tabela 4. Avaliação da frequência do consumo dos grupos alimentares pelos jogadores de League of legends (plataforma online) de Vitória da Conquista, Bahia, Brasil $(\mathrm{n}=28)$.

\begin{tabular}{|c|c|c|c|c|c|c|c|c|}
\hline \multirow[t]{2}{*}{ Grupo alimentares } & \multicolumn{2}{|c|}{ Diário } & \multicolumn{2}{|c|}{ Semanal } & \multicolumn{2}{|c|}{$\begin{array}{c}\text { Quinzenal/ } \\
\text { mensal }\end{array}$} & \multicolumn{2}{|c|}{$\begin{array}{l}\text { Eventual/ } \\
\text { Nunca }\end{array}$} \\
\hline & $\mathrm{n}$ & $\%$ & $\mathrm{n}$ & $\%$ & $\mathrm{n}$ & $\%$ & $\mathrm{n}$ & $\%$ \\
\hline Laticínios & 26 & 92,86 & 2 & 7,14 & 0 & - & 0 & - \\
\hline Pães, massas e cereais & 28 & 100 & 0 & - & 0 & - & 0 & - \\
\hline Tubérculos & 4 & 14,29 & 19 & 67,86 & 5 & 17,86 & 0 & - \\
\hline Leguminosas & 22 & 78,57 & 4 & 14,29 & 1 & 3,57 & 1 & 3,57 \\
\hline Ovos, carnes e peixes & 27 & 96,67 & 1 & 3,33 & 0 & - & 0 & - \\
\hline Frutas & 6 & 21,43 & 17 & 61 & 5 & 17,86 & 0 & - \\
\hline Castanhas e amêndoas & 0 & - & 2 & 7,14 & 11 & 39,29 & 15 & 53,57 \\
\hline Óleos e gorduras & 20 & 71,43 & 6 & 21 & 2 & 7,14 & 0 & - \\
\hline Açúcares e doces & 18 & 64,29 & 7 & 25 & 3 & 11 & 0 & - \\
\hline Bebidas e infusões & 26 & 92,86 & 2 & 7,14 & 0 & - & 0 & - \\
\hline
\end{tabular}

Discussão

As variações apresentadas pelos jogadores, em relação a idade $(21,53 \pm 2,74$ anos), altura $(176,63 \pm 0,063 \mathrm{~cm})$, peso $(79,26$ $\pm 19.95 \mathrm{~kg}), \operatorname{IMC}\left(25,31 \pm 6,04 \mathrm{~kg} / \mathrm{m}^{2}\right)$, massa gorda $(14,88 \pm 8,32 \mathrm{~kg})$, massa magra $(64,42 \pm$ $13,5 \mathrm{~kg})$ e $\operatorname{AMB}\left(54,63 \pm 16,5 \mathrm{~cm}^{2}\right)$, revela heterogeneidade no perfil da amostra na região de Vitória da Conquista, o que demonstra que se trata de um esporte sem um padrão especifico de físico especifico. Por se tratar de um esporte eletrônico seu direcionamento é nas habilidades cognitivas, como em alguns esportes coletivos e individuais. Vale ressaltar que se trata do 
primeiro estudo envolvendo esse tipo de esporte sobre essa abordagem e por isso a dificuldade de comparação com a literatura sobre o mesmo.

A média do IMC da amostra foi caracterizado como sobrepeso. De acordo com a literatura é um estado de risco aumentado para o desenvolvimento de doenças relacionadas a obesidade. Segundo Porto e colaboradores ${ }^{23}$, tem crescido o número de adultos jovens com o excesso de peso e risco aumentado de desenvolvimento de doenças crônicas não transmissíveis, como a hipertensão arterial sistêmica e diabetes mellitus, podendo ainda acarretar ao desenvolvimento de doenças cardiovasculares e complicações metabólicas.

O percentual de gordura apresentado pelos atletas teve a média $(17,96 \pm 0,72 \%)$ classificada como moderamente alto (17 a 20\%). Contudo, ao avaliar os atletas individualmente, foi observado que mais de $50 \%$ dos jogadores estavam classificados com percentual de gordura muito bom, bom e excelente, de acordo com a referência utilizada $^{18}$. Este resultado pode ser explicado pela adoção de um estilo de vida mais ativo por alguns dos jogadores.

Ao analisar o gasto calórico diário dos jogadores é possível observar um gasto energético médio de 2206,9 $\pm 390 \mathrm{Kcal}$, com uma ingestão diária média de 1834,2 \pm $7732,4 \mathrm{kcal} / \mathrm{dia}$, indicando que os jogadores estão consumindo menor quantidade que o necessário. Em relação aos macronutrientes, a média percentual de consumo diário de proteína, carboidrato e lipídio foram de 17,8\%,
$44,2 \%$ e $38,9 \%$ do gasto calórico total, respectivamente. Segundo a $\mathrm{AMDR}^{21}$ apenas o consumo proteico está dentro do preconizado, enquanto isso os valores de carboidratos estão abaixo das recomendações e os valores de lipídios acima.

Considerando a avaliação da frequência de consumo dos grupos alimentares pelos jogadores (tabela 4), 100\% dos participantes consomem pães, massas e cereais, $92,86 \%$ laticínios e 64,29\% açúcares e doces diariamente, os quais são fontes de carboidratos. Entretanto, é possível visualizar que mesmo que haja uma alta porcentagem da frequência de consumo diário desses grupos alimentares entre os participantes, não há o consumo de quantidades satisfatórias capazes de atenderem as recomendações diárias.

Baixas quantidades de carboidrato na dieta estão associadas ao aumento do consumo dos outros macronutrientes podendo levar a cardápios hiperproteicos e hiperlipídicos ${ }^{24}$. No presente estudo houve o aumento do consumo de lipídios.

Os micronutrientes analisados foram escolhidos de acordo com a atividade desempenhada pelos jogadores, a vitamina $\mathrm{A}$ relacionada a visão, as vitaminas do complexo $\mathrm{B}, \mathrm{C}$ e $\mathrm{E}$ devido, principalmente, pelas suas capacidades antioxidantes e vasodilatadoras, os minerais zinco, magnésio e selênio, pelas suas ações neuronais, já o ferro, sódio e fósforo, para analisar o perfil nutricional mais especifico desse público ${ }^{25}$. 
Os valores encontrados apresentam alguns percentuais de adequação fora da faixa média indicada, de 70 a $120 \%$ pela $\mathrm{RDA}^{20}$, sendo eles sódio, magnésio, fósforo e as vitaminas $\mathrm{C}, \mathrm{B} 3, \mathrm{~B} 6, \mathrm{~B} 9$ e B12 com valores de $154,01 \%, 43,18 \%, 58,92 \%, 47,11 \%, 8,13 \%$, $69,23 \%, 54,80 \%$ e $62,5 \%$, respectivamente.

Em relação ao sódio, o valor médio ingerido foi de $2310,2 \pm 716 \mathrm{mg}$, ultrapassando o valor recomendado segundo as DRIs. Valores elevados de sódio estão associados com o aumento do consumo de alimentos industrializados, assim como pontua Bielemann e colaboradore ${ }^{26}$ em sua pesquisa ao analisar a alimentação de jovens adultos da cidade de Pelotas - Rio Grande do Sul, observou a associação do alto consumo de alimentos industrializados e a elevada ingestão de sódio.

Tendo em consideração a frequência alimentar, os jogadores apresentaram baixa ingestão de tubérculos, frutas, castanhas e amêndoas. Segundo o Guia Alimentar da População Brasileira ${ }^{27}$ o consumo do grupo dos tubérculos deve ser estimulado por serem fontes de carboidratos e fibras e, no caso de algumas variedades, também de minerais e vitaminas, como o potássio e as vitaminas A e C.

Quanto o consumo de frutas a literatura $^{28}$ demonstra a associação entre $\mathrm{o}$ consumo desse grupo alimentar e à redução de incidências de fatores de risco para doenças cardiovasculares (DCV). Da mesma forma, a Organização Mundial de $\operatorname{Saúde}^{29}$ considerou que o consumo de cerca de 400g/dia (5 porções/dia) possui efeitos benéficos na incidência das DCV.

No estudo em questão houve a ausência do consumo de castanhas e amêndoas, sabe-se que este é um grupo alimentar que oferece inúmeros benefícios para a saúde, contudo o custo para aquisição destes alimentos é mais alto, o que pode ter sido um possível fator limitante para o consumo habitual pelos jogadores. Segundo Pereira, Fassina e Adami ${ }^{30}$ esse grupo alimentar é rico em fibras, vitaminas e minerais com propriedades anticancerígenas e antioxidantes, desempenhando papel importante nos processos.

\section{O presente estudo teve como} fragilidade a elevada quantidade de desistência por parte dos jogadores durante a etapa da realização dos registros alimentares, o que dificultou a exploração dos dados e a análise mais fidedigna do perfil nutricional e alimentar desses jogadores. Ainda, a falta de estudos semelhantes dificultou a comparação e discussão dos resultados.

\section{Conclusão}

Foi identificado o perfil de sobrepeso nos jogadores de league of legends da região de Vitória da Conquista, e uma alimentação com baixo consumo de tubérculos, frutas, castanhas e amêndoas, além do desbalanço no consumo de proteína, carboidrato e lipídeos. Ademais, o consumo dos micronutrientes magnésio, ferro, selênio, fósforo e as vitamina E, C, B1, B3, B6, B9 e B12 estavam abaixo dos recomendados. 
Os resultados demonstram que os jogadores possuem um perfil de risco ao desenvolvimento de DCNTs, contudo, este é o primeiro estudo na área envolvendo esse tipo de jogador voltado ao estado nutricional, dessa forma, é pertinente mais pesquisas nesse campo a fim de se traçar um perfil mais fidedigno desses jogadores para que assim, seja possível elaborar intervenções tanto educacionais quanto nutricionais caso necessário.

3. Ferrari S. From Generative to Conventional Play: MOBA and League of Legends. In: Digra Conference. 2013.

4. Funk, D.C.; Pizzo, A.D.; Baker, B.J. e-Sport management: Embracinge Sport education and research opportunities. Sport Management Review. 2018; 21 (1):7-13.

5. Saraiva PAC.E-sports: um fenômeno da cultura digital contemporânea. Lisboa: Instituto Universitário de Lisboa (ISCTE), 2013. Dissertação de Mestrado.

6. Rivera IR, Silva MAM, Silva RATA, Oliveira BAV, Carvalho ACC. Atividade física, horas de assistência à TV e composição corporal em crianças e adolescentes. Arq Brasileiro de Cardiologia. 2010; 95(2).

7. Smith-Menezes A, Duarte MFS, Silva RJS. Inatividade física, comportamento sedentário e excesso de peso corporal associados à condição socioeconômica em jovens. Rev Brasileira de Educação Física e Esporte. 2012; 26(3):411418.

8. Santos MG, Pegoraro M, Sandrini F, Macuco EC. Fatores de risco no desenvolvimento da aterosclerose na infância e adolescência. Arq

\section{Referências}

1. Barboza E, Silva A. A evolução tecnológica dos jogos eletrônicos: do videogame para o News game. $5^{\circ}$ Simpósio Internacional de Ciber jornalismo, 2014.

2. Jensen L. E-Sports: profissionalização e espetacularização em competições eletrônicas. Curitiba: Universidade Federal do Paraná, 2017.Dissertação de Mestrado.

Brasileiro de Cardiologia. São Paulo. 2008; 90(4):301-308.

9. Gonçalves ECA, Rinaldi W, Nunes HEG, Capdesboscq MC, Silva DAS. Baixos níveis de atividade física em servidores públicos do sul do Brasil: associação com fatores sociodemográficos, hipercolesterolemia e diabetes. Rev Andaluza de Med Del Deporte. 2017; 10(2):54-59.

10. Oliveira TC, Silva AAM, Santos CJN, Silva JS, Conceição IO. Atividade física e sedentarismo em escolares da rede pública e privada de ensino em São Luís. Rev de Saúde Pública. 2010; 44(6):996-1004.

11. Freire RS, Lélis FLO, Filho JAF, Nepomuceno MO, Silveira MF. Prática regular de atividade física: estudo de base populacional no Norte de Minas Gerais, Brasil. Rev Brasileira de Med do Esporte. 2014; 20(5): 345349.

12. E-SPORTV. League of Legends: como funciona o cenário competitivo do jogo. Disponível em: < https//portv.globo.com/site/esportv/noticia/league-of-legends-comofunciona-o-cenario-competitivo-dojogo.ghtml>. Acesso em: 18 de maio de 2021. 
13. E-SPORTV. Mundial de LoL 2019 tem pico de 44 milhões de espectadores simultâneos: veja balanço da Riot. Disponível em: < https://globoesporte.globo.com/e-

sportv/lol/noticia/mundial-de-lol-2019-tempico-de44-milhoes-de-espectadoressimultaneos-veja-balanco-da-riot.ghtml >. Acesso em: 18 de maio de 2021.

14. Brasil. Ministério da Saúde. Vigilância alimentar e nutricional - Sisvan: orientações básicas para a coleta, processamento, análise de dados e informação em serviços de saúde. Brasília. Ministério da Saúde. 2004.

15. World Health Organization. Obesity. Preventing and managing the global epidemic. Reportof a WHO consultationonobesity. WHO/NUT/NCD/981, WHO, Geneva. 1998.

16. Durnin, JVGA, Wormersley J. Body fatassessed from total Body densityand its estimation from skin fold thickness: measurements on 481 men and womenaged from 16 to 72 years. British Journal of Nutrition. 1974; 32: 77-79.

17. Siri WE. Body composition from fluids paces and density: analysis of methods. In: Brozek J. E.; Henschel, A. Technique for measuring body composition. Washington. National Academyof Science. 1961; 223-244.

18. Pollock ML, Wilmore JH. Exercícios na saúde e na doença: avaliação e prescrição para prevenção e reabilitação. Medsi: Rio de Janeiro 1993. 734p.

19. Gurney JM, Jelliffe DB. Arm anthropometry in nutritional assessment: nomogram for rapid calculation of muscle circumference and cross-sectional muscle and fat areas. Am J Clin Nutr 1973; 26:912- 5.
20. Food and Agriculture Organization. Human Energy Requirements. Reportof a Joint FAO/WHO/UNU. Expert Consultation. FAO TechnicalReport Series 1. Rome. 2004.

21. Institute of Medicine (IOM). Dietary Reference Intakes for energy, carbohydrate, fiber, fat, fattyacids, cholesterol, protein, and amino acids (macronutrients). Washington. DC. National Academies Press. 2002.

22. Instituteof Medicine (IOM). Dietary Reference Intakes for Vitamin A, Vitamin K, Arsenic, Boron, Chromium, Copper, Iodine, Iron, Manganese, Molybdenum, Nickel, Silicon, Vanadium, andZinc. Washington. DC. National Academies Press. 2001.

23. Porto TNRS, Cardoso CLR, Baldoino LS, Martins VS, Alcântara SML, Carvalho DP. Prevalência do excesso de peso e fatores de risco para obesidade em adultos.Rev Eletrônica Acervo Saúde. 2019; 22:308.

24. Silva AF, Santos VS. Qualidade nutricional de dietas da moda veiculadas em revistas não científicas. Rev de Saúde e Ciências Biológicas. 2021; 9 (1): 1-5.

25. Mahan, LK; Raymond JL. Krause Alimentos, Nutrição e Dietoterapia. $14^{\mathrm{a}}$ ed. Rio de Janeiro: Elsevier; 2018.

26. Bielemann RM, Motta JVS, Minten GC, Horta BL, Gigante DP. Consumo de alimentos ultraprocessados e impacto na dieta de adultos jovens. Rev de Saúde Pública. 2015; 49 (28).

27. Brasil, Ministério da Saúde. Guia Alimentar para a População Brasileira. - Promovendo a alimentação saudável. Brasília: Ministério da Saúde, 2014.

28. Gan Y, Tong X, Li L, Cao S, Yin, X, Gao C et al. Consumption of fruit and vegetable and 
risk of coronary heart disease: A meta-analysis of prospective cohort studies. International Journal of Cardiology. 2015; 183:129-137.

29. World Health Organization (WHO). Global strategyon diet, physical activity and health. Geneva. 2004.

30. Pereira ALD, Fassani P, Adami FS. Benefícios para a saúde associados ao consumo de oleaginosas. Rev Uningá. 2016; 50: 82-85.

Declaração de conflitos de interesse - Nós autores declaramos que não há conflitos de interesse.
Reservado aos Editores

Dara da Submissão 05/04/2021

Data da aprovação 01/06/2021 